\title{
Peritoneal resident macrophages in mice with MLL-AF9-induced acute myeloid leukemia show an M2-like phenotype
}

\author{
Chong Chen ${ }^{1,2,3,4}$, Rong Wang ${ }^{1}$, Wenli Feng ${ }^{1}$, Feifei Yang ${ }^{1}$, Lina Wang ${ }^{1}$, Xiao Yang ${ }^{1}$, Li Ren ${ }^{2,3,4}$, \\ Guoguang Zheng ${ }^{1}$
}

${ }^{1}$ State Key Laboratory of Experimental Hematology, National Clinical Research Center for Blood Diseases, Institute of Hematology \& Blood Diseases Hospital, Chinese Academy of Medical Sciences \& Peking Union Medical College, Tianjin, China; ${ }^{2}$ Department of Clinical Laboratory, Tianjin Medical University Cancer Institute and Hospital, National Clinical Research Center for Cancer, Tianjin, China; ${ }^{3}$ Key Laboratory of Cancer Prevention and Therapy, Tianjin, China; ${ }^{4}$ Tianjin's Clinical Research Center for Cancer; National Human Genetic Resources Sharing Service Platform Tianjin, Tianjin, China

Contributions: (I) Conception and design: G Zheng; (II) Administrative support: L Ren; (III) Provision of study materials or patients: C Chen, R Wang, W Feng, F Yang; (IV) Collection and assembly of data: C Chen, L Wang; (V) Data analysis and interpretation: C Chen, L Wang, X Yang; (VI) Manuscript writing: All authors; (VII) Final approval of manuscript: All authors.

Correspondence to: Guoguang Zheng. State Key Laboratory of Experimental Hematology, Institute of Hematology and Blood Diseases Hospital, Chinese Academy of Medical Sciences and Peking Union Medical College, Tianjin, China Email: zhenggg@ihcams.ac.cn; Li Ren. Department of Clinical Laboratory, Tianjin Medical University Cancer Institute and Hospital, National Clinical Research Center for Cancer, Key Laboratory of Cancer Prevention and Therapy, Tianjin's Clinical Research Center for Cancer, National Human Genetic Resources Sharing Service Platform, Tianjin, China. Email: roland-li@163.com.

Background: Acute myeloid leukemia (AML) is a devastating disease with a poor prognosis. Innate and adaptive immunity is closely related to the progression of leukemia. Macrophages within the leukemic microenvironment have a tendency toward a leukemia-permissive phenotype. However, the characteristics of macrophages in leukemia, including their kinetics, gene expression, and functional roles have not been fully illuminated.

Methods: In the current study, the characteristics of peritoneal resident macrophages, which were large peritoneal macrophages (LPM), from mice with mixed lineage leukemia (MLL)-AF9-induced AML were investigated. AML-associated large macrophages (AML-LPM) were gated as F4/80 ${ }^{\text {high }}$ MHC-II' by flow cytometry. To further investigate the relationship between the leukemic microenvironment and macrophage characteristics, RNA sequencing was performed. Meanwhile, apoptosis, killing ability, and phagocytic function of peritoneal resident macrophages in MLL-AF9-induced AML were assessed.

Results: The results suggested that AML microenvironment was found to affect the kinetics and morphology of peritoneal resident macrophages. The results of RNA sequencing suggested that the gene expression of AML-LPMs differed significantly from that of normal LPMs. The AML microenvironment also had effects on the apoptosis, killing ability, and phagocytic function of peritoneal resident macrophages.

Conclusions: These data suggest that peritoneal resident macrophages in mice with AML induced by MLL-AF9 show an M2-like phenotype. The reversal of macrophage polarization in the leukemic microenvironment may potentially enhance the immunotherapeutic effect in AML.

Keywords: Peritoneal resident macrophages; large peritoneal macrophages (LPM); leukemic microenvironment; MLL-AF9-induced acute myeloid leukemia; M2-like phenotype

Submitted Dec 17, 2020. Accepted for publication Jan 26, 2021.

doi: $10.21037 /$ atm-21-139

View this article at: http://dx.doi.org/10.21037/atm-21-139 


\section{Introduction}

Macrophages are immune cells that can be found in almost every organ and tissue in the body (1). Macrophages present as different phenotypes and play diverse roles depending on the organ or tissue, and the phenotype and function were regulated by microenvironmental signals (2). Gene expression characteristics analysis of macrophages in a steady state revealed heterogeneity among macrophages in different tissues. Significant differences in gene expression patterns existed among the four classical macrophage populations, which was closely related to heterogeneity in organ-specific microenvironments (3). Macrophages enter into body tissue in their undifferentiated form and differentiate into resident macrophages. Macrophages in different tissues show significant differences in morphology, phenotype, and function, and heterogeneity also exists among macrophages in the same tissue microenvironment (4). Studies suggest that there are two monocyte subsets in the blood, which have distinct functions and might differentiate into different types of macrophages (5).

Different subsets of resident macrophages exist in certain tissues and serosal cavities, such as the lung, spleen, and abdominal cavity (6). In the spleen, at least three types of macrophage subsets can be found: red medulla macrophages, metalloid macrophages, and limbic macrophages. Meanwhile, in the peritoneal cavity, two types of macrophages are present, small peritoneal macrophages (SPM) and large peritoneal macrophages (LPM), both of which are peritoneal resident macrophages (5). These two macrophage subgroups are involved in the fight against pathogens related to intraperitoneal infection, as well as inflammation resist and the maintenance of intraperitoneal homeostasis. The SPM and LPM subgroups differ from other intraperitoneal cells. Both have been found to express the typical molecular markers CD11b and F4/80, but at different levels (5). F4/80 is expressed at much higher levels in LPMs than in SPMs. MHC-II-type molecules can be found at high levels in SPMs but are not expressed in LPMs. SPMs and LPMs are derived from different sources. SPMs are derived from hematopoietic stem cells and are supplemented by circulating monocytes, while LPMs are derived from precursor cells of the yolk sac, have no relation to hematopoietic precursor cells, and can self-renew. SPMs and LPMs show differences in function due to their different origins and phenotypes. In Lavin et al.'s study, neither SPMs nor LPMs could produce a large number of pro-inflammatory or anti-inflammatory cytokines under normal physiological conditions (7). In acute inflammatory states [such as lipopolysaccharide (LPS) abdominal stimulation], most SPMs secrete cytokines such as nitric oxide (NO), tumor necrosis factor (TNF- $\alpha$ ), macrophage inflammatory protein $1 \alpha$ (MIP-1 $1 \alpha$ ), and regulated upon activation normal $\mathrm{T}$ cell expressed and secreted factor (RANTES). LPMs are also able to secrete cytokines such as keratinocyte chemoattractant $(\mathrm{KC})$, granulocyte colonystimulating factor (G-CSF), and Monocyte chemotactic protein-1 (MCP-1) (5). SPMs and LPMs play different roles in inflammation, with SPMs having more M1-type macrophage properties in inflammatory infection and LPMs being more like M2-type macrophages (7). Recent studies have shown that LPMs can induce B-1 cells to secrete IgA through the GALT-independent pathway, and that LPMs are resupplied to the abdominal cavity during inflammation and participate in the process of inflammatory regression (8). Macrophages play a key role in the host anti-infection process and are able to absorb and degrade microorganisms. Both SPMs and LPMs display phagocytic function, albeit with some differences. Studies have shown that LPMs have significantly higher phagocytic function than SPMs. SPMs and LPMs play an important role in maintenance of intra-abdominal homeostasis, antiinfection/inflammation, phagocytosis, and other processes.

Most macrophages are derived from hematopoietic stem cells; however, some originate from early embryonic stem cells. Educated macrophages in tissue microenvironments are known as tissue-specific macrophages (4). Tissue-specific macrophages show heterogeneity in different organs and tissues, and heterogeneity even exists among macrophages in the same tissue (9). Macrophages in the same tissue are also heterogeneous in different pathologic microenvironments. Phenotypically, educated resident macrophages show great variation, depending on the microenvironment. Phenotypic and functional heterogeneity has reportedly been found in macrophages in the blood system, nerve tissue, connective tissue, and glomerular mesangial tissue; however, little attention has been paid to peritoneal macrophage heterogeneity (7). To date, there have been few reports about the heterogeneity of peritoneal macrophages and no studies on the characteristics of peritoneal resident macrophages in leukemia. Therefore, in the present study, the dynamics and functions of peritoneal macrophages in different physiological subsets and peritoneal macrophage polarization were explored in mice with acute myeloid leukemia (AML). We present the following article in 
accordance with the ARRIVE reporting checklist (available at http://dx.doi.org/10.21037/atm-21-139).

\section{Methods}

\section{Mice and AML model}

Male C57BL/6J mice (6 to 8 weeks old) were supplied by the Animal Centre of the Institute of Hematology and Blood Diseases Hospital. All mice were maintained in a certified, specific-pathogen-free animal facility. All experiments involving animals were performed under a project license (No.: EK2020065) granted by the Animal Care and Use Committee of the institution, in compliance with institutional guidelines for the care and use of animals.

\section{MLL-AF9-induced AML model}

An MLL-AF9-induced mouse AML model was established as previously described (10). Briefly, lineage-negative and Sca-1-positive (Lin-Sca- ${ }^{+}$) cells were obtained from the bone marrow (BM) of normal C57BL/6J mice and transfected with retrovirus carrying the MLL-AF9 fusion gene (MSCV-MLL-AF9-IRES-GFP). Then, the infected cells were transplanted into lethally irradiated C57BL/6J mice. All mice developed AML. In this study, nonirradiated AML mouse model was used. A non-irradiated AML mouse model was established by injecting leukemia cells into the caudal vein of non-irradiated C57BL/6 mice $\left(1 \times 10^{6}\right.$ cells/mouse $)$. All mice developed AML. All mice were sacrificed, and peritoneal washes were performed for further analysis.

\section{Cell preparation of peritoneal washes}

Peritoneal cells were obtained by peritoneal washing following standard protocol (11). The cells were resuspended in RPMI 1640 Medium (GibcoTM, Invitrogen Corporation, France) for further analysis.

\section{Flow cytometric analysis and cell sorting}

Cells of different macrophage subpopulations were obtained through cell sorting using a FACSCanto II flow cytometer (BD Biosciences), and then stained with antibodies against mouse F4/80 (APC conjugated, BM8, BioLegend) and against MHC-II (PE-conjugated, AF6-120.1, BioLegend).

Peritoneal cells from normal mice were prepared as a negative control. Peritoneal cells were washed and resuspended in perlsucht bacillenemulsion (PBE) containing $0.5 \%$ fetal bovine serum (FBS) and were used to fluorescence activated cell sorting (FACS) analysis. Data were analyzed with FlowJo software (version 7.6.1). LPMs were gated as $\mathrm{F} 4 / 80^{\text {high }} \mathrm{MHC}^{-} \mathrm{II}^{-}$, and SPMs were gated as F4/80 ${ }^{\text {low }} \mathrm{MHC}^{-\mathrm{II}^{+}}$.

\section{Wright-Giemsa staining}

LPMs were spun to slides and standard Wright-Giemsa staining was performed. The morphological characteristics of the LPMs were analyzed using a light microscope (AXIO Observer A1, ZEISS, Germany).

\section{Cell cycle analysis}

The cell cycles of peritoneal resident macrophages from leukemic and normal mice were analyzed through propidium iodide staining following standard protocol (10). Briefly, cells were fixed with ice-cold $70 \%$ ethanol and permeabilized for 11 hours. Meanwhile, RNase was used to disintegrate RNA. Finally, propidium iodide dye was added before analysis on FACS.

\section{cDNA synthesis and real time polymerase chain reaction}

Cells were lysed, and total RNA was extracted using the RNeasy Mini Kit (Qiagen, Valencia, CA). Reverse transcription was executed using a Super Script FirstStrand Synthesis System (Invitrogen, Carlsbad, CA). Realtime polymerase chain reaction (PCR) was carried out on the Step One real-time PCR system (Applied Biosystems, Foster City, CA). The $2^{-\Delta \Delta \mathrm{Ct}}$ method $[\Delta \Delta \mathrm{Ct}=(\mathrm{CtTARGET}$ - CtGAPDH $)_{\text {sample }}$ - $\left.(\text { CtTARGET - CtGAPDH })_{\text {calibrator }}\right]$ was used to compare the expression levels of target genes. All primers of target genes are listed as previously described (11).

\section{$R N A$ sequencing}

LPMs from the AML microenvironment were defined as AML-LPMs, while those from the normal physiological microenvironment were defined as normal LPMs. SPMs and LPMs were sorted from peritoneal washes on day 15 after the injection of leukemia cells, and they were also obtained from normal mice. RNA sequencing was performed by the Beijing Genomics Institute using 
BGISEQ-500 following the standard protocol.

\section{Statistical analysis}

All results were described as the mean \pm standard deviation (SD). Comparisons between two groups were analyzed by unpaired Student's $t$-test, while multiple comparisons were performed using analysis of variance. Statistical differences were considered significant when $\mathrm{P}<0.05$.

\section{Results}

\section{Acquisition of AML-LPMs}

In this study, an AML model induced by MLL-AF9 was successfully constructed (Figure 1A). The AML onset process is divided into three phases according to time after transplantation: the early phase ( 7 days after transplantation), metaphase (15 days after transplantation), and late phase (23 days after transplantation) (10). Changes in the levels of $\mathrm{GFP}^{+}$leukemia cells in the bone marrow and abdominal cavity of the mice during these phases are shown in Figure 1B. We found a high level of leukemia cell invasion in the peritoneal cavity, which might have impacted peritoneal resident macrophages (Figure 1C).

\section{The AML microenvironment affected the kinetics and morphology of LPMs}

With the progression of leukemia, the number of SPMs in the abdominal cavity firstly increased and then decreased, while the number of LPMs showed the opposite pattern. Neutrophils are also involved in AML progression, and the number of $\mathrm{F} 4 / 80^{\text {low }}$ neutrophils first increased and then decreased. In the AML model, the number of LPMs in the metaphase was increased (Figure 2A). A morphological comparison with sorted leukemia cells was performed through Wright-Giemsa staining. We found that the vacuolation of LPMs occurred in the middle and late stages of AML onset, and the proportion of LPMs increased with leukemia progression (Figure 2B).

\section{AML microenvironment affects LPM gene expression}

To further investigate the effects of different microenvironments on macrophages, the gene expression profiles of LPMs from two groups were analyzed. The results suggested that gene expression of LPMs in the leukemic microenvironment differed significantly from that of normal LPMs. Differentially expressed genes (DEGs; with fold change $(\mathrm{FC}) \geq 2$, false discovery rate (FDR) $\leq 0.001$ ) were used to further confirm our observation. A total of 1,532 differentially expressed genes were found in AML, including 907 up-regulated genes and 625 downregulated genes (Figure $3 A, B$ ). To explore the distinctive features of AML-LPMs, the 1532 DEGs were subjected to Kyoto Encyclopedia of Genes and Genomes (KEGG) pathway analysis. Figure $3 C$ shows the top 15 enriched KEGG pathways; of note, these included the cell cycle and p53 signaling pathways. Gene Ontology (GO) analysis of the DEGs in AML was also performed. The top 10 significantly enriched GO entries are listed in Figure 3D. The results showed that in terms of biological processes, the DEGs were mainly related to the cell cycle. In terms of cellular components, there were changes in chromosomal components and cellular components. In terms of molecular functions, the DEGs were mainly related to binding, especially protein binding (Figure 3D). Between AML-LPMs and normal LPMs, common DEGs included arginine1 (ARG1), cluster of differentiation 206 (CD206), inducible nitric oxide synthase (iNOS), interleukin-6 (IL-6), TNF- $\alpha$, interleukin-10 (IL-10), interleukin-1 $\beta$ (IL-1 $\beta$ ), and transforming growth factor- $\beta$ (TGF- $\beta$ ). The results of verification by reverse transcription PCR were consistent with those of RNA sequencing (Figure $3 E$ ).

\section{AML microenvironment affects the immunologic function of LPMs}

A nitric oxide fluorescent probe was used to detect the active nitrogen content of LPMs in different microenvironments, comparing the killing ability of macrophages. The active nitrogen content of AML-LPMs was significantly lower than that of normal LPMs, which was different from the gene expression of iNOS (Figure 4A). Macrophages in different microenvironments were used to phagocytize FITC-GFP ${ }^{+}$particles; it was found that FITC-GFP ${ }^{\text {medium }}$ was the main type of AML-LPM, but FITC-GFP ${ }^{\text {low }}$ and FITC-GFP ${ }^{\text {high }}$ were the main types of normal LPMs (Figure 4B). There were significant differences in phagocytic function between AML-LPMs and normal LPMs. Propidium iodide staining revealed more G2/M/S phase cells among AML-LPMs, showing that AML-LPMs have the potential for proliferation; however, normal LPMs did not have this function (Figure 4C). 
A MSCVMLL-AFMRESGFP

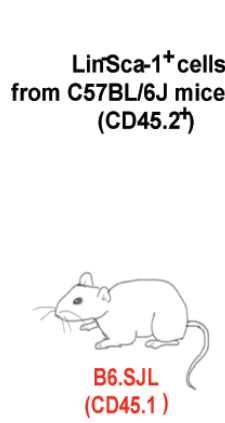

B
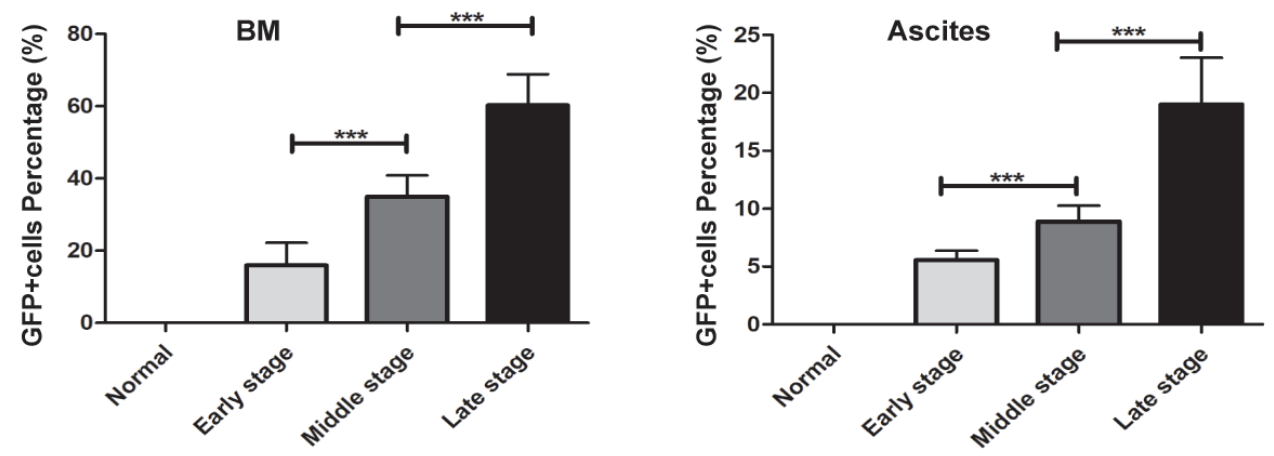

C
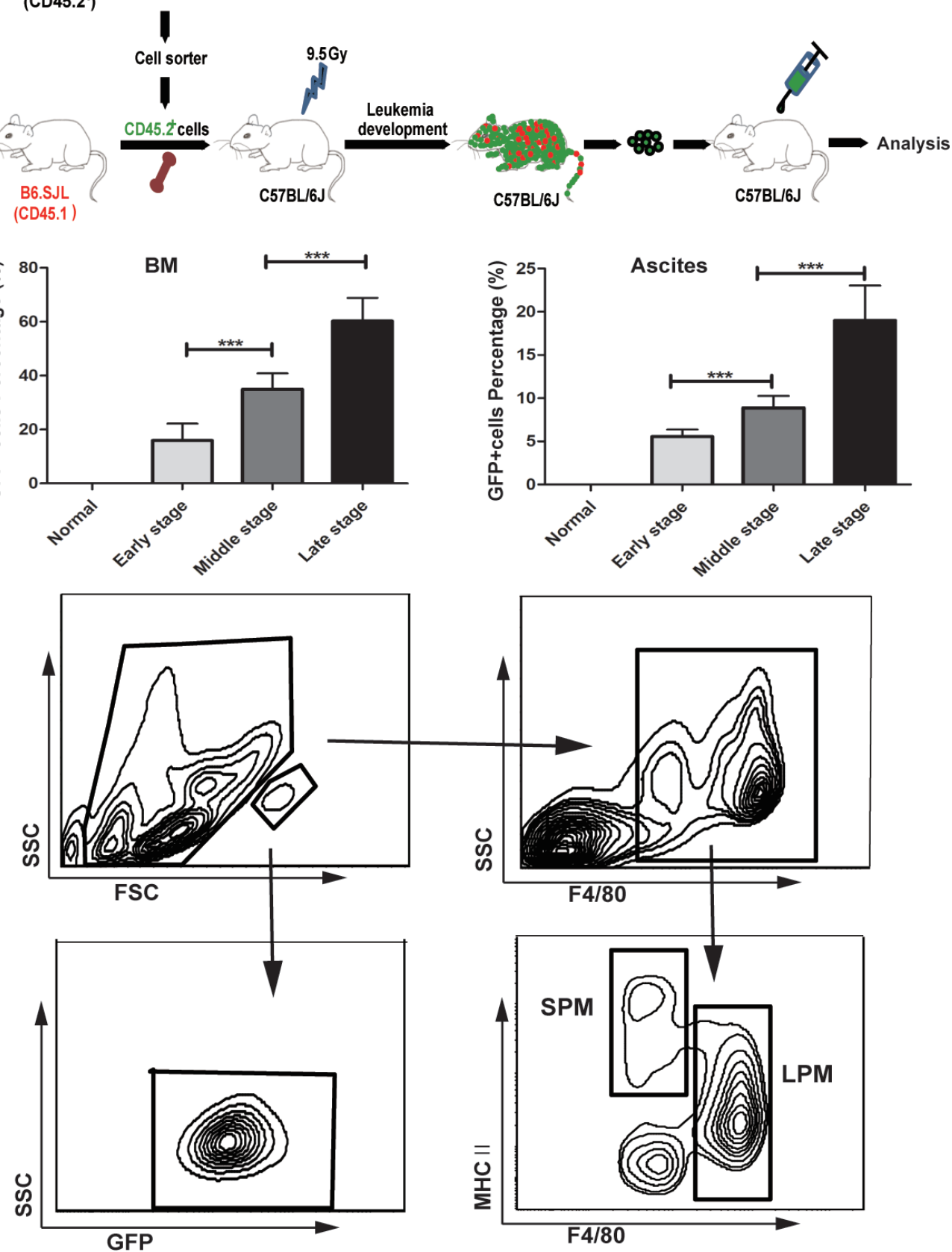

Figure 1 Isolation of LPMs (large peritoneal macrophages) in the MLL-AF9-induced AML model. (A) Schematic diagram of the MLLAF9-induced AML model construction; (B) changes in GFP+ leukemic cells during the pathogenesis of MLL-AF9-induced AML (acute myelogenous leukemia); (C) AML-LPMs were obtained using a flow-sorting technique. ${ }^{* * *}, \mathrm{P}<0.001$. 
A

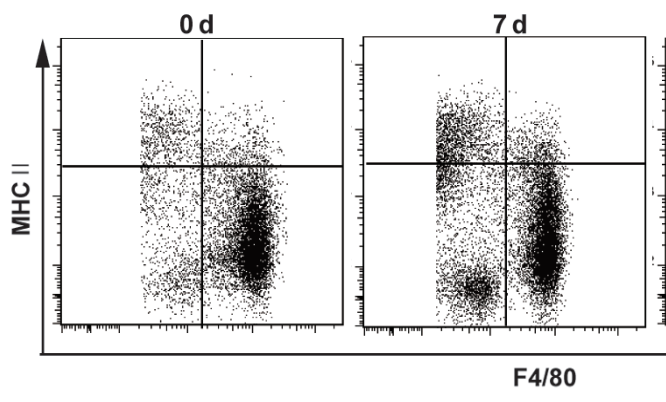

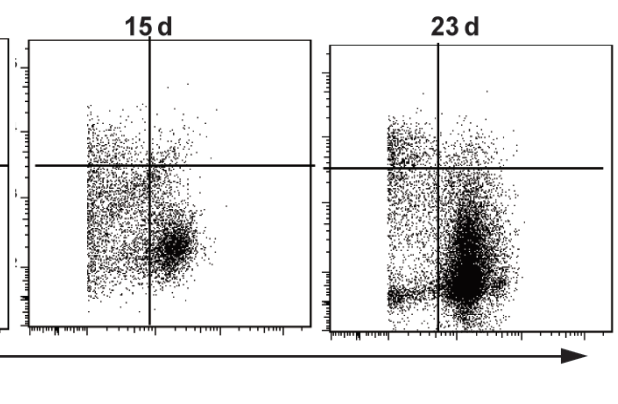
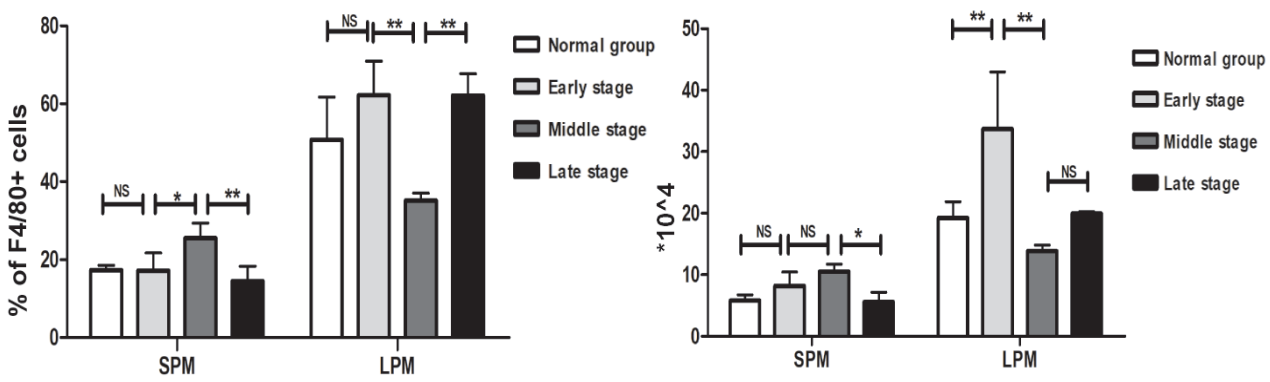

B

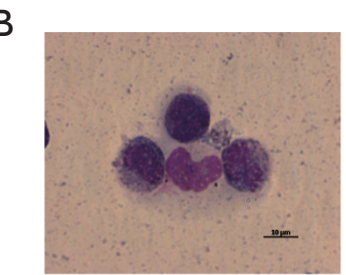

Normal LPM

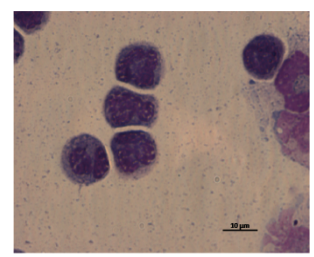

Early stage

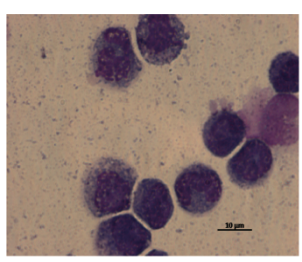

Middle stage

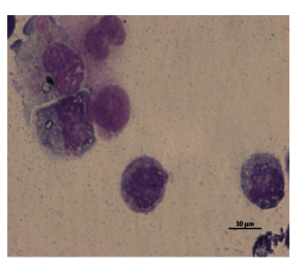

Late stage

Figure 2 The kinetics and morphology of peritoneal resident macrophages in mice with MLL-AF9-induced AML. (A) The changes in each subgroup of F4/80+ cells in MLL-AF9-induced AML mice; (B) the morphological characteristics of Wright-Giemsa-stained LPMs from different stages of leukemia onset. *, $\mathrm{P}<0.05$; **, $\mathrm{P}<0.01$. MLL, mixed lineage leukemia; AML, acute myeloid leukemia; LPMs, large peritoneal macrophages.

\section{Discussion}

The peritoneal cavity selectively attracts and maintains specialized immune cells such as macrophages, which influence the immune microenvironment of the peritoneal cavity (12). There is some phenotypic and functional evidence demonstrating that the two distinct subsets of macrophages show significant heterogeneity in the mouse peritoneal cavity (5). For instance, they differ in size, as determined by flow cytometric light-scatter measurements. Previous research revealed that SPMs and LPMs in the peritoneal cavity of mice can express F4/80 and CD11b, which had a phagocytic function in vivo. However, the present study found there to be significant differences in the expression of several surface markers between SPMs and LPMs, especially in the expression of MHC-II, which is expressed by SPMs but not by LPMs. Significant differences were also found in the expression of F4/80, which is highly expressed by LPMs but not SPMs. Under steady state conditions, LPMs, which originate independently from hematopoietic precursors, maintain their physiological numbers and retain their ability to proliferate in the peritoneal cavity of mice; conversely, under inflammatory conditions, SPMs, which appear to originate from circulating monocytes, increase in number remarkably (13). Yang et al. reported that leukemia cell proliferation led to the aggravation of microenvironment deterioration (14). In the leukemic microenvironment, LPMs could self-renew and maintained the dynamic balance of cell proliferation in the abdominal cavity, and peaked in number during the early stage. At the same time, the leukemic microenvironment affected the morphology of LPMs. Moreover, studies have 
A

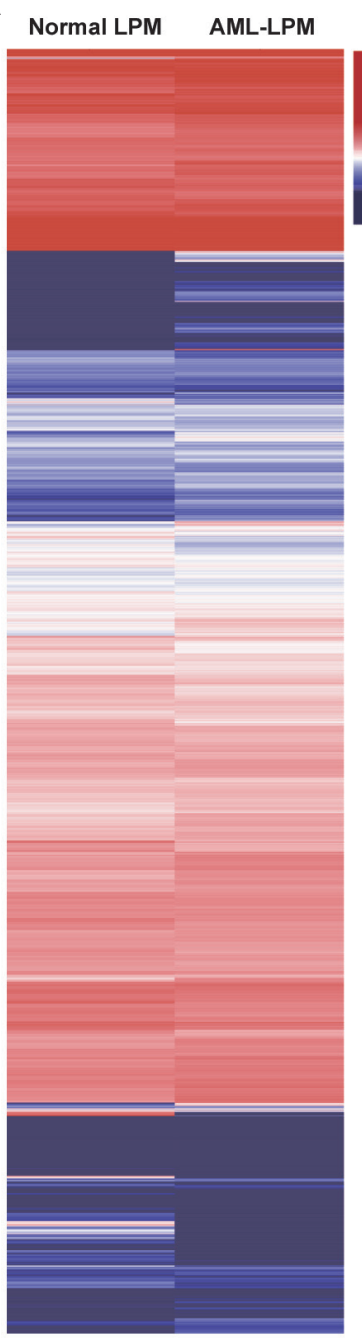

B

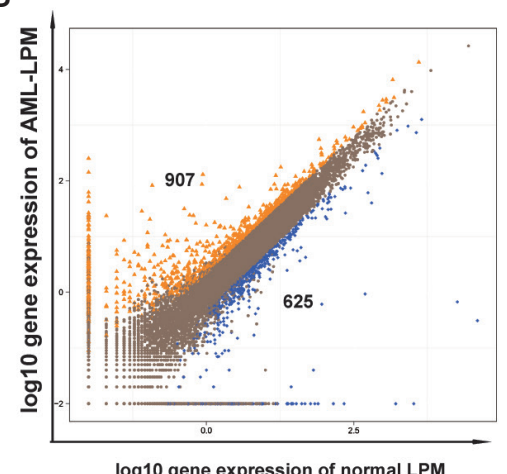

C

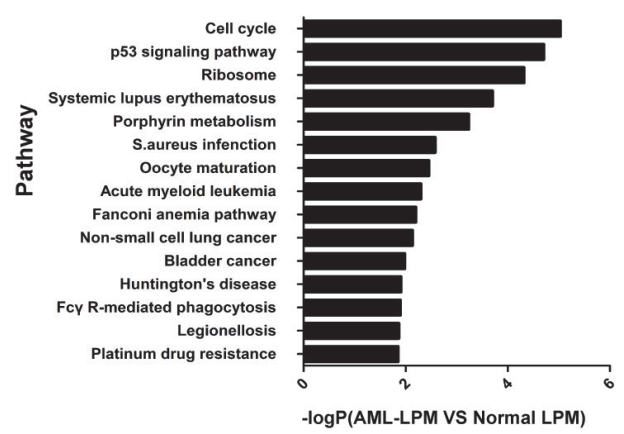

D
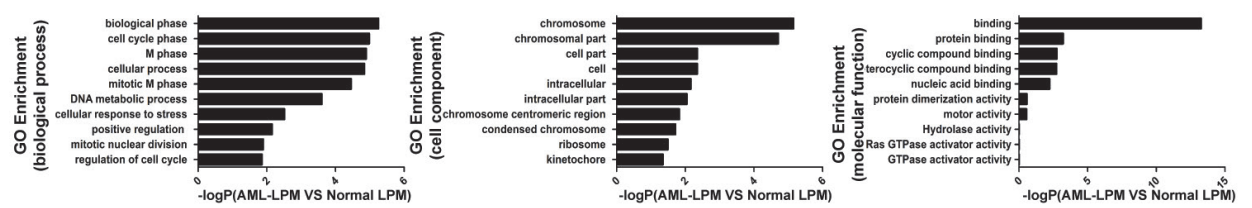

E
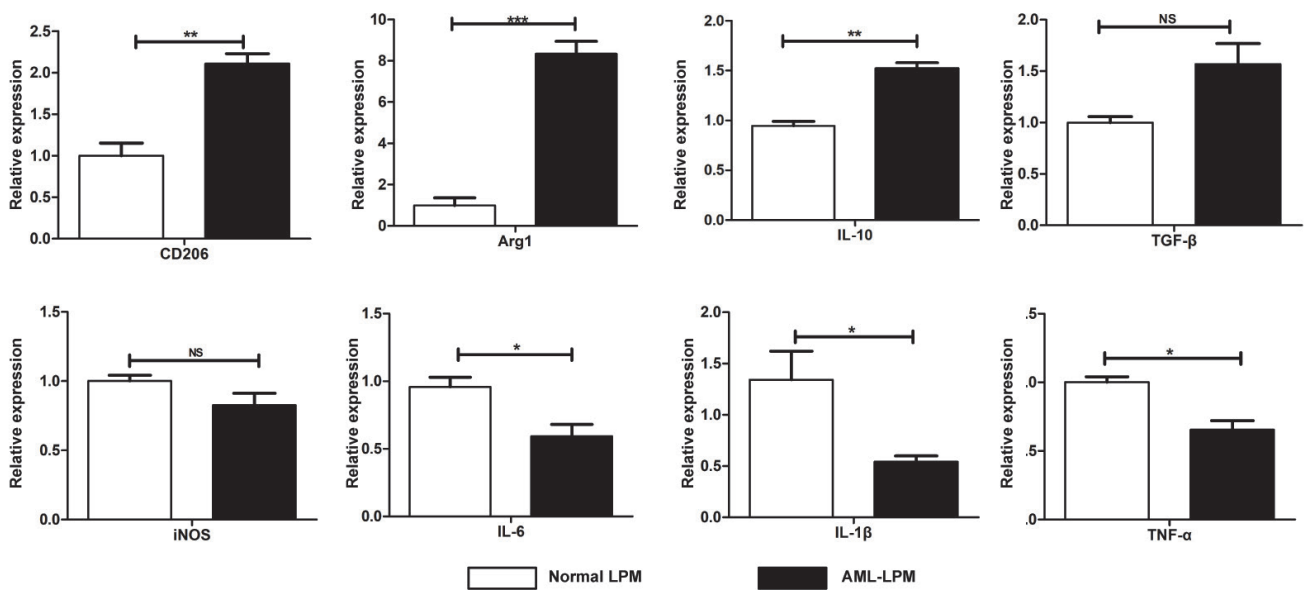

Figure 3 Effects of different microenvironments on the gene expression profiles of peritoneal resident macrophages. (A) Hierarchical clustering of gene expression between AML-LPMs (acute myelogenous leukemia associated large peritoneal macrophages) and normal LPMs; (B) number of differentially expressed genes (DEGs; normal LPMs or AML-LPMs, FC $\geq 2$, FDR $\leq 0.001$ ); (C) top 15 pathways in Kyoto Encyclopedia of Genes and Genomes analysis of non-overlapping DEGs; (D) top 10 terms in Gene Ontology analysis of nonoverlapping DEGs; (E) DEGs in macrophages verified by reverse transcription-polymerase chain reaction (RT-PCR). All data in columns are shown as mean \pm standard deviation $(\mathrm{SD}) .{ }^{*}, \mathrm{P}<0.05 ;{ }^{* *}, \mathrm{P}<0.01{ }^{* * *}, \mathrm{P}<0.001$.

shown that macrophage subsets also affect other peritoneal cell populations such as T cells and dendritic cells directly, which may play an important role in the immune state of the peritoneal cavity $(5,15)$.

AML-LPMs and LPMs were respectively analyzed using KEGG pathway analysis, and the results showed that although macrophages that were educated by the leukemic microenvironment shared common characteristics, such as the molecular function of binding, educated macrophages exhibited significant differences, including in changes in cellular composition and biological processes. In most solid tumors, the majority of tumor-associated macrophages (TAMs) are M2-like macrophages. In this paper, we found that AML-LPMs expressed M2-associated genes, thus differing from normal LPMs. As a malignant tumor of the blood system, leukemia has many similarities with solid tumors in pathogenesis but has its own unique characteristics $(9,16)$. As in polarized M2 macrophages, 

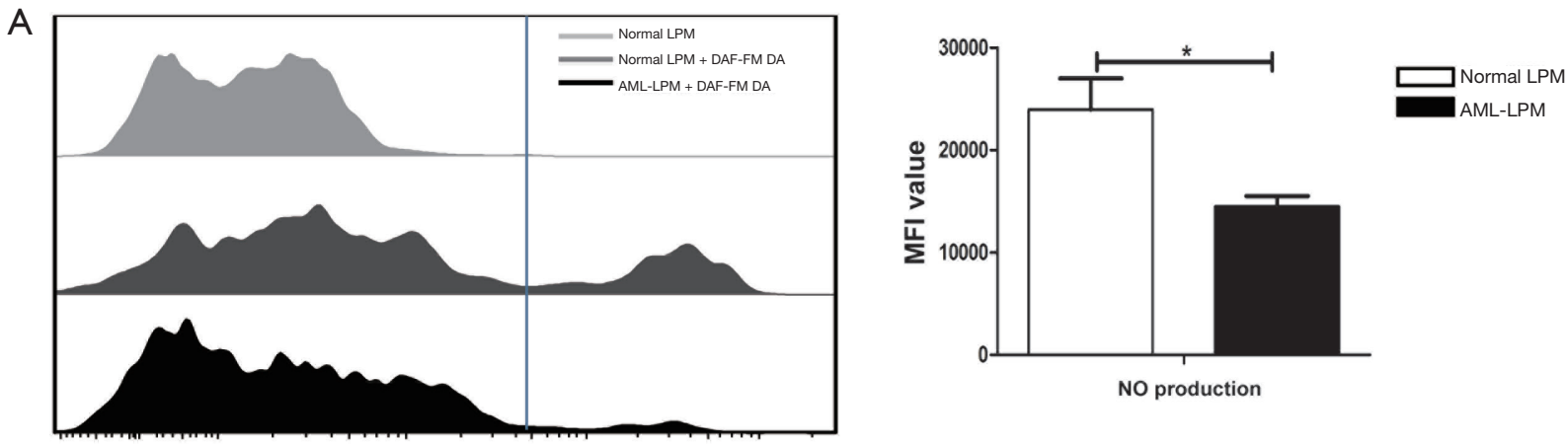

B
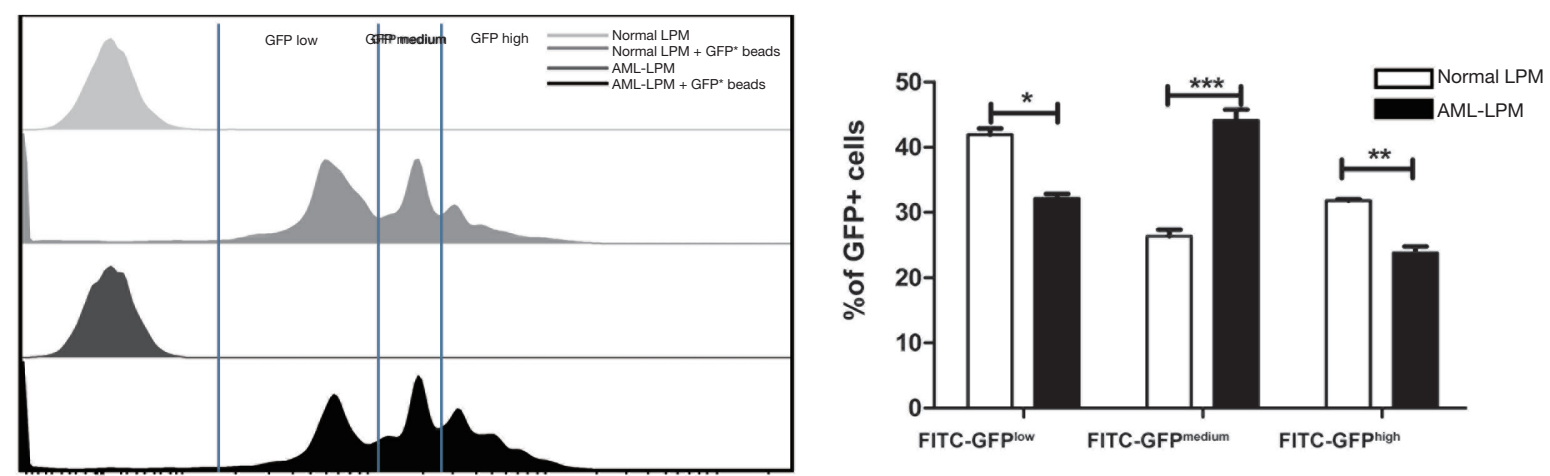

C
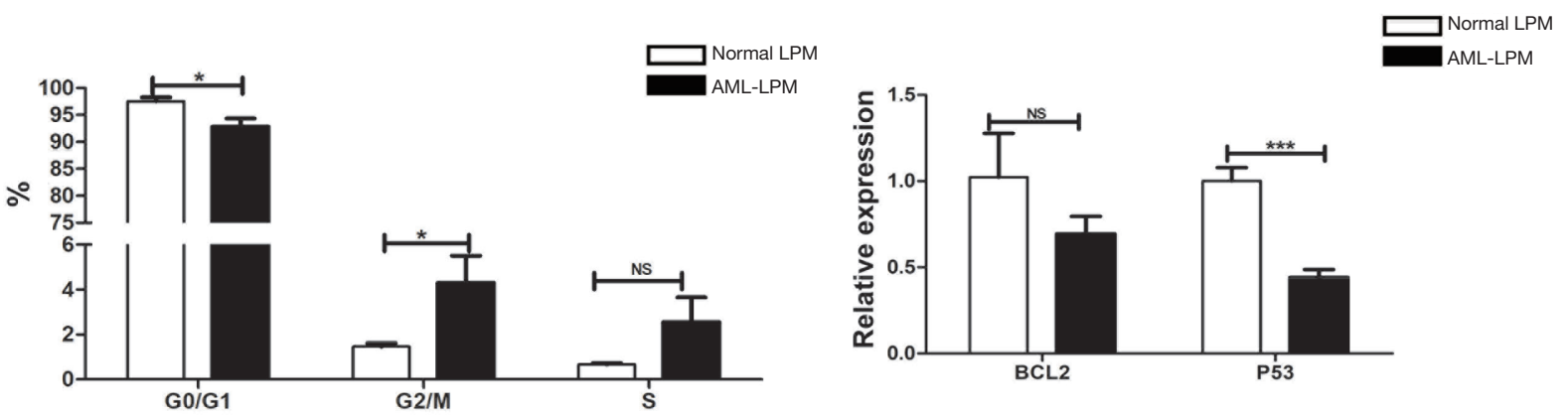

Figure 4 Effects of different microenvironments on the functional roles of peritoneal resident macrophages. (A) Effects of different microenvironments on the killing ability of peritoneal resident macrophages; (B) effects of different microenvironments on the phagocytic function of peritoneal resident macrophages; (C) effects of different microenvironments on the apoptosis of peritoneal resident macrophages. *, $\mathrm{P}<0.05 ;{ }^{* *}, \mathrm{P}<0.01 ;{ }^{* * *}, \mathrm{P}<0.001$.

anti-inflammatory molecules, such as IL-10, arginase-1, and mannose (CD206), were expressed at high levels in AML-LPMs; however, unlike M2-polarized macrophages, they did not significantly express transforming growth factor- $\beta$ (TGF- $\beta$ ). M2-polarized macrophages have been confirmed to perform an immunosuppressive role in vivo (17). Al-Matary et al. revealed that the number of M2-like TAMs was remarkably increased in the BM of AML patients compared to normal controls (18). Some results have suggested that TAMs perform a crucial role in leukemia progression. Researchers also found that TAMs were polarized to an M2-like phenotype in bone marrow by the leukemic microenvironment, which subsequently played an immunosuppressive role in the $\mathrm{BM}$ and spleens of leukemia-bearing mice $(17,18)$. Conversely, bone marrow-derived macrophages (BMDMs) from leukemia sustained the in vitro proliferation of AML cells, which is apparently higher than that of heathy mice $(18,19)$. LPMs also interact with the leukemia microenvironment to accelerate disease progression. A recent study showed 
that an increase in M2-like TAMs was associated with poor prognosis in patients with AML (14). Some genes, such as M2-like markers including CD206 and IL-10, which were also studied in pancreatic cancer (20), have been confirmed to be highly expressed in AML-LPMs. Differences in cytokine expression between different pathological microenvironments may be an important factor contributing to the heterogeneity of TAMs $(20,21)$.

Macrophages play an important role in pathogenic bacteria killing and phagocytosis. Nitric oxide secreted by macrophages, is involved in killing and inhibiting exogenous microorganisms. Intracellular NO from macrophages is synthesized through the NOS pathway and regulated by iNOS gene, and its intracellular NO content is closely related to the anti-inflammatory and killing functions of macrophages (22). NO synthesis can be induced by IFN- $\gamma$, and this induction process often requires the presence of a second signal (TNF- $\alpha$ or LPS), using IFN- $\gamma$ or TNF- $\alpha$ alone to induce only low doses of $\mathrm{NO}$ with $\mathrm{NO}$ direct cytotoxic effects $(8,23,24)$. Studies have shown that in the AML microenvironment, there was significant difference in intracellular NO content between AML-LPMs and normal LPMs, in other words there had some difference in killing function (Figure 4A). These may be caused by immunosuppression, which is closely related to immune reprogramming of immune cells. Immunosuppression is related to the effect of TAMs on other immune cells, such as $\mathrm{T}$ cells, through different signaling pathways, which results in the formation of a pro-tumorigenic microenvironment (25). Comparison of macrophage phagocytosis showed that AML-LPM and LPM phagocytic $\mathrm{GFP}^{+}$particles also showed significant differences, which may be attributable to significant differences in the innate immune-related functions of macrophages in different microenvironments. CD47, which is expressed on macrophages, acts as an inhibitor in phagocytosis (25). Anti-CD47 antibody-based approaches may enhance phagocytosis of leukemia cells by LPMs (26). Comparison of cell cycles of different LPMs between leukemic and normal microenvironment showed cell proportion had significant differences in G0/G1 and G2/M stages, with more LPM proliferation observed in the leukemic microenvironment, which was also consistent with the results of RNA sequencing. The expression level of the proapoptotic gene P53 was higher in normal LPMs than in AML-LPMs, while that of the anti-apoptotic BCL2 gene was not significantly different between the two groups.

In conclusion, the heterogeneity of macrophages resident in the same tissue is closely related to different pathological microenvironments (27). Leukemia is similar to malignant solid tumors but has some differences. During the progression of leukemia, changes in the microenvironment of M2-like TAMs may improve their killing and phagocytic innate immune functions $(28,29)$. Changing the polarization state of leukemia-associated macrophages is expected to be a new immunotherapeutic strategy to treat leukemia.

\section{Acknowledgments}

Funding: This study was supported by the Science \& Technology Development Fund of Tianjin Education Commission for Higher Education (project number: 2018KJ048, to CC).

\section{Footnote}

Reporting Checklist: The authors have completed the ARRIVE reporting checklist. Available at http://dx.doi. org/10.21037/atm-21-139

Data Sharing Statement: Available at http://dx.doi. org/10.21037/atm-21-139

Conflicts of Interest: All authors have completed the ICMJE uniform disclosure form (available at http://dx.doi. org/10.21037/atm-21-139). The authors have no conflicts of interest to declare.

Ethical Statement: The authors are accountable for all aspects of the work in ensuring that questions related to the accuracy or integrity of any part of the work are appropriately investigated and resolved. All experiments involving animals were performed under a project license (No.: EK2020065) granted by the Animal Care and Use Committee of the institution, in compliance with institutional guidelines for the care and use of animals.

Open Access Statement: This is an Open Access article distributed in accordance with the Creative Commons Attribution-NonCommercial-NoDerivs 4.0 International License (CC BY-NC-ND 4.0), which permits the noncommercial replication and distribution of the article with the strict proviso that no changes or edits are made and the original work is properly cited (including links to both the formal publication through the relevant DOI and the license). See: https://creativecommons.org/licenses/by-nc-nd/4.0/. 


\section{References}

1. Epelman S, Lavine KJ, Randolph GJ. Origin and functions of tissue macrophages. Immunity 2014;41:21-35.

2. Quail DF, Joyce JA. Microenvironmental regulation of tumor progression and metastasis. Nat Med 2013;19:1423-37.

3. Wynn TA, Chawla A, Pollard JW. Macrophage biology in development, homeostasis and disease. Nature 2013;496:445-55.

4. Gordon S, Pluddemann A, Martinez Estrada F. Macrophage heterogeneity in tissues: phenotypic diversity and functions. Immunol Rev 2014;262:36-55.

5. Ghosn EE, Cassado AA, Govoni GR et al. Two physically, functionally, and developmentally distinct peritoneal macrophage subsets. Proc Natl Acad Sci U S A 2010;107:2568-73.

6. Davies LC, Taylor PR. Tissue-resident macrophages: then and now. Immunology 2015;144:541-8.

7. Lavin Y, Mortha A, Rahman A, et al. Regulation of macrophage development and function in peripheral tissues. Nat Rev Immunol 2015;15:731-44.

8. Sica A, Mantovani A. Macrophage plasticity and polarization: in vivo veritas. J Clin Invest 2012;122:787-95.

9. Cai L, Michelakos T, Deshpande V, et al. Role of TumorAssociated Macrophages in the Clinical Course of Pancreatic Neuroendocrine Tumors (PanNETs). Clin Cancer Res 2019;25:2644-55.

10. Yang F, Wang R, Feng W, et al. Characteristics of NK cells from leukemic microenvironment in MLLAF9 induced acute myeloid leukemia. Mol Immunol 2018;93:68-78.

11. Chen S, Yang X, Feng W, et al. Characterization of peritoneal leukemia-associated macrophages in Notch1induced mouse $\mathrm{T}$ cell acute lymphoblastic leukemia. Mol Immunol 2017;81:35-41.

12. Petty AJ, Yang Y. Tumor-associated macrophages in hematologic malignancies: new insights and targeted therapies. Cells 2019;8:1526.

13. Park MY, Kim HS, Jeong YS, et al. Novel Sca$1(+)$ macrophages modulate the pathogenic progress of endotoxemia. Biochem Biophys Res Commun 2020;533:83-9.

14. Yang X, Feng W, Wang R, et al. Hepatic leukemiaassociated macrophages exhibit a pro-inflammatory phenotype in Notch1-induced acute T cell leukemia. Immunobiology 2018;223:73-80.

15. Wang R, Feng W, Wang $H$, et al. Blocking migration of regulatory $\mathrm{T}$ cells to leukemic hematopoietic microenvironment delays disease progression in mouse leukemia model. Cancer Lett 2020;469:151-61.

16. Hohtari H, Bruck O, Blom S, et al. Immune cell constitution in bone marrow microenvironment predicts outcome in adult ALL. Leukemia 2019;33:1570-82.

17. Feng W, Yang X, Wang L, et al. P2X7 promotes the progression of MLL-AF9 induced acute myeloid leukemia by upregulation of $\mathrm{Pbx} 3$. Haematologica 2020. [Epub ahead of print]. doi: 10.3324/haematol.2019.243360.

18. Al-Matary YS, Botezatu L, Opalka B, et al. Acute myeloid leukemia cells polarize macrophages towards a leukemia supporting state in a Growth factor independence 1 dependent manner. Haematologica 2016;101:1216-27.

19. Giannoni P, Pietra G, Travaini G, et al. Chronic lymphocytic leukemia nurse-like cells express hepatocyte growth factor receptor (c-MET) and indoleamine 2,3-dioxygenase and display features of immunosuppressive type 2 skewed macrophages. Haematologica 2014;99:1078-87.

20. Schulz A, Durr C, Zenz T, et al. Lenalidomide reduces survival of chronic lymphocytic leukemia cells in primary cocultures by altering the myeloid microenvironment. Blood 2013;121:2503-11.

21. Yang F, Feng W, Wang H, et al. Monocyte-Derived Leukemia-Associated Macrophages Facilitate Extramedullary Distribution of T-cell Acute Lymphoblastic Leukemia Cells. Cancer Res 2020;80:3677-91.

22. Liang JJ, Liu YF, Ng TK, et al. Peritoneal macrophages attenuate retinal ganglion cell survival and neurite outgrowth. Neural Regen Res 2021;16:1121-6.

23. Qian BZ, Pollard JW. Macrophage diversity enhances tumor progression and metastasis. Cell 2010;141:39-51.

24. Caner A, Asik E, Ozpolat B. SRC Signaling in Cancer and Tumor Microenvironment. Adv Exp Med Biol 2021;1270:57-71.

25. Johnson LDS, Banerjee S, Kruglov O, et al. Targeting CD47 in Sezary syndrome with SIRPalphaFc. Blood Adv 2019;3:1145-53.

26. Feng M, Jiang W, Kim BYS, et al. Phagocytosis checkpoints as new targets for cancer immunotherapy. Nat Rev Cancer 2019;19:568-86.

27. Cassado AA, D'Imperio Lima MR, Bortoluci KR. Revisiting mouse peritoneal macrophages: heterogeneity, development, and function. Front Immunol 2015;6:225.

28. Zhao S, Mi Y, Guan B, et al. Tumor-derived exosomal miR-934 induces macrophage M2 polarization to promote 
liver metastasis of colorectal cancer. J Hematol Oncol 2020;13:156.

29. Daley D, Mani VR, Mohan N, et al. Dectin 1 activation on macrophages by galectin 9 promotes pancreatic carcinoma and peritumoral immune tolerance. Nat Med 2017;23:556-67.

(English Language Editor: J. Reynolds)

Cite this article as: Chen C, Wang R, Feng W, Yang F, Wang L, Yang X, Ren L, Zheng G. Peritoneal resident macrophages in mice with MLL-AF9-induced acute myeloid leukemia show an M2-like phenotype. Ann Transl Med 2021;9(3):266. doi: 10.21037/atm-21-139 